\title{
Effectiveness of Aromatherapy Treatment in Alleviating Fatigue and Promoting Relaxation of Mothers during the Early Postpartum Period
}

\author{
Kyoko Asazawa' ${ }^{1}$, Yoshihiro Kato ${ }^{1}$, Ryosuke Koinuma1 ${ }^{1}$, Nao Takemoto ${ }^{1}$, Shiho Tsutsui ${ }^{2}$ \\ ${ }^{1}$ Department of Nursing, Tokyo Healthcare University, Tokyo, Japan \\ ${ }^{2}$ Tokyo Medical Center, Tokyo, Japan \\ Email: 11DN001x2U-ts@slcn.ac.jp
}

How to cite this paper: Asazawa, K., Kato, Y., Koinuma, R., Takemoto, N. and Tsutsui, S. (2018) Effectiveness of Aromatherapy Treatment in Alleviating Fatigue and Promoting Relaxation of Mothers during the Early Postpartum Period. Open Journal of Nursing, 8, 196-209.

https://doi.org/10.4236/ojn.2018.83017

Received: February 17, 2018

Accepted: March 25, 2018

Published: March 28, 2018

Copyright $\odot 2018$ by authors and Scientific Research Publishing Inc. This work is licensed under the Creative Commons Attribution International License (CC BY 4.0).

http://creativecommons.org/licenses/by/4.0/

(c) $\underset{\mathrm{EY}}{\mathrm{C}}$ Open Access

\begin{abstract}
Background: This study aimed to determine the effectiveness of aroma hand treatment in alleviating fatigue and promoting relaxation of mothers in their early postpartum period. Methods: This is a quasi-experimental study with a two-group pretest-post-test design which used purposive sampling and non-random assignment of 242 consenting Japanese early postpartum mothers who were patients from a hospital maternity unit in Tokyo, Japan. The intervention involved a 20-minute aroma hand treatment using an effleurage method. The participants selected from 5 essential oils: pure lavender, ylang-ylang, citron, rosewood, and sweet orange. Relaxation promotion and fatigue alleviation as the main outcomes were measured using self-administered valid and reliable questionnaires. Statistical analysis was performed using the two-sample t-test, two-way factorial ANOVA, and simple main effect test. SPSS ver. 23.0 (SPSS, Chicago, IL, USA) was used for data analysis at a 5\% significance level. Results: Of 242 participants (intervention group, $\mathrm{n}=122$; comparison group, $\mathrm{n}=120$ ), 229 participants (intervention group, $\mathrm{n}=115$; comparison group, $\mathrm{n}=114$ ) were analyzed per protocol. The intervention group showed a significant improvement in relaxation $(t=6.43, p<0.001)$. A significant difference in the simple main effect test of relaxation $(\mathrm{F}=37.58, \mathrm{p}$ $<0.001)$ was found. However, there was no significant reduction in fatigue. The majority (88.4\%) of the participants indicated high satisfaction with the aroma hand treatment, and $90.5 \%$ evaluated the length of intervention time as appropriate. Conclusions: The aroma hand treatment effectively promoted relaxation of early postpartum mothers, but was less effective in alleviating their fatigue. Based on their high satisfaction rate, aroma hand treatment using essential oils may be considered effective in promoting relaxation of early postpartum mothers.
\end{abstract}




\section{Keywords}

Aromatherapy, Postpartum Period, Fatigue, Relaxation

\section{Introduction}

Postpartum mothers face difficult periods after giving birth and have unmet needs for support in those periods, particularly support from other new mothers [1]. Moreover, postpartum mothers spend stressful periods from sleep deprivation, sleep disturbance, and fatigue [2]. In particular, mothers feel fatigue from delivery and experience growing fatigue from breastfeeding and infant care early in their postpartum period [3]. Fatigue and lack of energy have been accepted as common effects of childbirth in the postpartum period, with fatigue causing mothers to experience negative feelings and become uncomfortable [4]. Although postpartum mothers also experience other physical conditions in addition to fatigue and physical exhaustion, such as sleep-related problems, pain, sex-related concerns, hemorrhoids, constipation, and breast problems, fatigue remains a major concern [3].

Approximately $60 \%$ of postpartum mothers have moderate fatigue, which is significantly associated with maternal health [5]. Fatigue in the early postpartum period is significantly related to and is a predictor of postpartum depression [6]. Therefore, to reduce the risk of maternity blues, it is important for early postpartum mothers to have relaxing experiences and fatigue reduction so that they can take care of their child with peace of mind.

The essential oils used in aromatherapy treatments promote relaxation when their fragrant component stimulates the hypothalamus and activates the parasympathetic nervous system [7]. Thus, relaxation promotion and fatigue reduction are expected from the fragrance of essential oils and their forearm application using an effleurage method.

In terms of the effects of using aromatherapy in the medical field, blood pressure and anxiety were reduced [8], as well as confused behavior in dementia patients [9]. Aromatherapy full-body massage for postpartum mothers induced a significant decrease in the maternity blues score, a decrease in the State-Anxiety Inventory score, and an increase in the Profile of Mood States [10]. A decrease in the anxiety score was associated with using aromatherapy slow-stroke back massage [11]. From the present survey results, aromatherapy treatments may be considered appropriate and helpful for postpartum mothers experiencing nervousness and fatigue.

We previously developed a short aroma hand treatment method for early postpartum mothers as a pilot study in preparation for the present study. The results of our previous pilot study indicated fatigue reduction and relaxation promotion of the mothers post intervention. The process evaluation in terms of mothers' satisfaction level and impression of providers' thoughtfulness con- 
firmed the usefulness of aroma hand treatment [12].

In the present study, we established a comparison group together with an optimum sample size to clarify the effects of using the method developed for the mothers in the intervention group. This study aimed to specifically examine the effects of a short aroma hand treatment for early postpartum mothers in terms of relaxation promotion and fatigue reduction, and the satisfaction level of the mothers undergoing the aromatherapy. The authors predicted a favorable outcome from the aroma hand treatment intervention with a high satisfaction.

\section{Methods}

\subsection{Study Design}

This study used a quasi-experimental design consisting of a convenience sample and a nonequivalent control group with non-random group assignment.

\subsection{Participants and Setting}

The participants were mothers in their early postpartum period (days $1-7$ ) and who had been admitted before delivery to a large-scale general hospital with a 52-bed maternity unit in Tokyo, Japan. The hospital had perinatal and life-saving transport support systems. The hospitalized mothers were in their early 30 s as average age. The ratio of cesarean sections was $27.7 \%$. Consistent with maternity care in Japan, the length of hospital stay was typically 4 - 5 days for a normal delivery and 8 days for a cesarean section [13]. The inclusion criteria were as follows: 1) after normal delivery on days 1 - 4 or repeat cesarean section on postoperative days $2-7,2$ ) fluent in Japanese, and 3) granted permission to participate by the primary physician. The exclusion criteria were patients with the following conditions: 1) emergency cesarean section, 2) severe psychiatric disorders, or 3) allergic reactions, reddening and eczema. The researchers together with the nurse manager confirmed the mothers in their early postpartum period who fulfilled the above-mentioned inclusion criteria using convenience sampling. The researchers asked these mothers who met the inclusion criteria to participate in the present research.

The approximate number of participants necessary for the study to have an adequate effect size was calculated using the estimation method developed by Cohen (1992) [14]. The intervention effect was calculated for a significance level of alpha $=0.05$, power $=0.80$, and effect size $\gamma=0.40$. Thus, the calculation formula indicated that the sample size should be 98 participants. Based on the authors' pilot study, a dropout rate of $14.7 \%$ was anticipated. Applying the results to Cohen's formula, the anticipated dropout rate yielded, $\mathrm{n} \geq 98 /(1-0.147)=$ 114. Therefore, the target sample size was set at 228 participants (114 for each group).

\subsection{Intervention}

The intervention treatment included applying 1 of 5 types of essential aroma oil 
(i.e., pure lavender, ylang-ylang, citron, rosewood, and sweet orange) by massaging to the mother's hands and forearms. The intervention treatment was provided by 10 research assistants. During their year-long preparation, they attended various training sessions and seminars provided by the Certified Nurses of Japanese Society of Aromatherapy and they gained experience through volunteer-related activities. In addition, the research assistants passed a technical qualifying examination for independent professional skills and completed a lecture on puerperium care. The aroma treatments used a blend of sweet almond oil as the carrier oil and the applied essential oil. The concentration of the essential oil was diluted to within $2 \%$ in consideration of the women who were in their early postpartum period [15].

Prior to the intervention treatment, the research assistants provided verbal and written explanations to the participants regarding the efficacy of the various aromas of the oils. The participants were then able to smell each oil, after which they selected the oil for their treatment. To ensure safety, the participants received a patch test before starting the intervention treatment to determine any allergic reaction to the aromatherapy ingredients.

The research assistants applied the essential oil using the massage method described by Hashemi et al. (2015) [16]. The intervention treatment consisted of using optimal effleurage as a basic technique to ensure that the oil on the palm spreads thoroughly across the entire palm, and then the participants' hands and forearms were slowly and gently stroked to promote the flow of blood and lymph. Treatment was started on the left side followed by the right side and was performed for approximately 20 minutes. Each participant received 1 aromatherapy treatment in a private room in either the sitting or lying down position. The room temperature was maintained at approximately $25^{\circ} \mathrm{C}$. The rationale for the treatment options was informed by the research assistants in relation to the time setting without causing burden on the massage [17] and to the treatment using effleurage [16].

\subsection{Procedures}

The authors recruited participants during their postpartum hospital stay using purposive sampling with the assistance of the hospital nurse manager. The authors and nurse manager confirmed whether the postpartum mothers met the inclusion criteria.

The authors informed the mothers verbally and in writing about the purpose of the study, as well as about the confidentiality, anonymity, and safety of personal data. If the mothers agreed to participate in the study, the authors obtained their written consent. The authors provided an explanation of their right to withdraw from the study without penalty, and a withdrawal form. The participants could also mail the withdrawal form if necessary. Data were collected from August 2016 to December 2016. We used an anonymous self-filled questionnaire and attempted to obtain reliable data by the following methods: 1) in the pilot study, the deficiencies in the methods were confirmed and the questionnaire was 
corrected; 2) the participants received a detailed explanation on how to fill out the questionnaire; 3 ) data with missing values and inadequacies were excluded.

The self-report questionnaires were administered during the mothers' hospital stay. Completed questionnaires were placed in a secure box on the unit, providing convenience and anonymity. Collection of the outcome evaluation data was carried out for the pre-test and post-test. The pre-test was requested the day before the intervention, whereas the post-test was requested for the current day after about 24 hours of the pre-test. The timing of the post-test immediately after the intervention was in accordance with the studies of Nakakita and Takenoue (2009) [17] and Imura et al. (2006) [10], and in consideration of the postpartum mothers breastfeeding schedule and child-rearing education classes.

\subsection{Ethical Considerations}

This study was conducted after obtaining approval from the Ethics Committee for Epidemiological Studies of Tokyo Healthcare University, Tokyo, Japan (approval no. 27-27) on December 28, 2015. A written explanation was provided to the participants regarding the study objectives, methods, protection of anonymity, and voluntary basis of participation following the Declaration of Helsinki. It was also explained that the collected data would be used solely for this study. A signed informed consent was obtained from each participant.

\subsection{Outcome Measures}

The demographics and outcome measures included fatigue and relaxation scales and author-developed outcome evaluation. The outcome measures provided both quantitative and qualitative data.

\subsubsection{Fatigue Scale}

Fatigue was assessed using the "subjective symptoms" 13-item subscale of the Self-Diagnosis Check List for Assessment of Worker's Accumulated Fatigue [18]. In Japan, this checklist is widely used for investigating mother's fatigue because of its convenient scaling and ease of providing a response. Although this checklist was constructed for a working population, it had been modified to 12 items after its validity was examined [19]. The reliability of the scale was established on the basis of the Cronbach's alpha coefficient range of $0.72-0.84$ [19]. Although the participants in previous studies were instructed to rate their psychological health during the past month, the participants in the present study were asked to consider their current symptoms. The scale consists of 12 items with 4 response categories ranging from 0 (no fatigue) to 3 (higher fatigue). Higher scores indicated higher fatigue. A score of 5 - 10 indicates a slightly higher fatigue, and a score of 11 and above indicates a higher fatigue. In the present study, the Cronbach's alpha coefficient of the fatigue scale was 0.86 .

\subsubsection{Relaxation Scale}

Relaxation was evaluated using a revised version of the rating scale of emotion using the items referring to relaxation. Nedate and Kamisato (1984) developed 
this scale for measuring the subjective sense of relaxation [20]. Takahashi (1996) revised the scale to increase item representation [21]. The relaxation scale is a 4-item inventory which includes the following items: "I feel laid-back", "I feel unclenched", "I feel relieved", and "I feel in a receptive mood". The response categories ranged from 0 (strongly disagree) to 10 (strongly agree). Higher scores indicated higher relaxation. Takahashi (1996) reported that the instrument had acceptable reliability, and the Cronbach's alpha coefficient range was 0.81 - 0.87. In the present study, the Cronbach's alpha coefficient of the relaxation scale was 0.83 .

\subsubsection{Process Evaluation}

For the process evaluation, the participants completed the 5-item survey developed by the authors which includes 1) treatment satisfaction, 2) meeting of expectations, 3) adequacy of intervention time, 4) satisfaction in receiving essential oils, and 5) provider's communication skills. A five-point Likert scale was used for scoring each item, with higher scores indicating a more positive acceptance of the intervention. The participants also responded to an open-ended question seeking their opinion about the intervention.

\subsection{Statistical Analysis}

SPSS ver. 23.0 (SPSS, Chicago, IL, USA) was used for data analyses with the significance level set at 5\%. Unpaired Student's t-test and $\chi 2$-test were used to compare the following basic characteristics: each scale score of the participants and each variable before the program between the 2 groups. Per-protocol analysis, two-way factorial analysis of variance (ANOVA), and simple main effect test were used to analyze and test the differences between the intervention and control groups to verify the effectiveness of the intervention. If significant differences were found in the two-way factorial ANOVA, a simple main effect test was used in the next stage. A frequency distribution table was created from the 5 items in the process evaluation. The open-ended remarks were analyzed using constant comparative analysis as described by Lyn (2015) [22].

\section{Results}

\subsection{Participant Attributes}

Regarding the flow of participants who met the study inclusion criteria (Figure 1), there were 140 mothers in the intervention group and 140 mothers in the comparison group.

Among these mothers, there were 122 mothers in the intervention group and 120 mothers in the comparison group who agreed to participate in the study. The study was completed by 115 mothers (94.3\%) in the intervention group and 114 mothers (95.0\%) in the comparison group.

Finally, the per-protocol analysis included 115 participants in the intervention group and 114 participants in the comparison group; dropouts such as those who lacked data, canceled, or did not return their responses were excluded. 


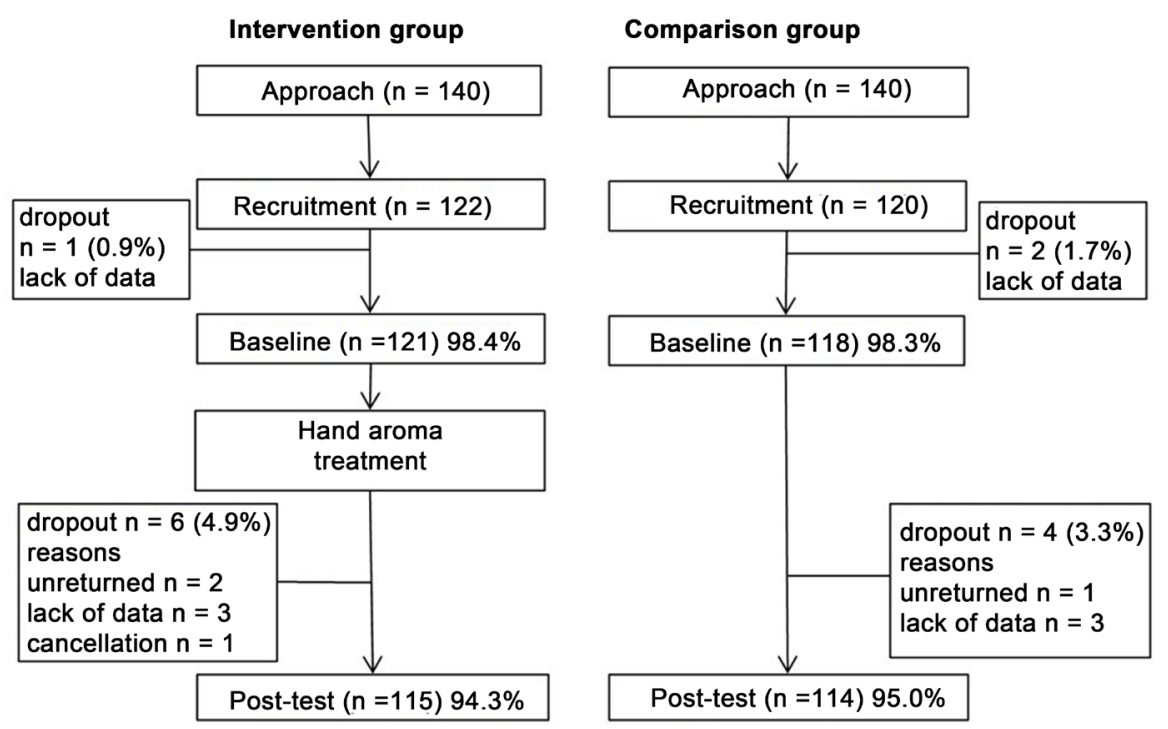

Figure 1. Flowchart of study participants $(\mathrm{N}=229)$.

There were no significant differences in the characteristics or pretest scale scores between the intervention and control groups (Table 1). The cesarean section was a repeat procedure. The previous diseases were Hashimoto's thyroiditis and Graves' disease. In addition, the pregnancy complications were pregnancy anemia, pregnancy-induced hypertension, and threatened premature delivery. The pretest average scores of each scale for all participants (intervention/comparison) were as follows: Fatigue, 8.7/8.6 $(\mathrm{t}=0.096, p=0.924)$; Relaxation, 22.7/22.9 $(\mathrm{t}=$ $-0.105, p=0.917)$.

\subsection{Verification of Interaction effects between Intervention and Time Using Two-Way Factorial ANOVA}

It was anticipated that there would be an interaction effect between intervention and time and a difference in the pattern of changes between the 2 groups. First, to examine a two-factor interaction, two-way factorial ANOVA was conducted for each scale. For the ANOVA, the dependent variable was each scale score, the between-subjects factor was the presence or absence of intervention, and the within-subjects factor was time. There were significant interaction effects between intervention and time in the Relaxation scale $(\mathrm{F}=39.68, p<0.001)$. However, there was no significant interaction effects between intervention and time in the Fatigue scale $(\mathrm{F}=3.71, p=0.055)$ (Table 2).

\subsection{Verification of Differences between Groups Using Simple Main Effect Test}

To verify the differences between groups, a simple main effect test for each scale with a significant interaction effect was performed. A significant difference between the intervention group and the comparison group was shown by the simple main effect test of Relaxation ( $\mathrm{F}=37.58, p<0.001$ ) (Figure 2). There was no significant difference between the groups in the simple main effect test of 
Table 1. Baseline comparison between the 2 groups $(\mathrm{N}=229)$.

\begin{tabular}{|c|c|c|c|c|c|c|c|}
\hline \multirow[b]{3}{*}{ Demographic variable } & \multirow{2}{*}{\multicolumn{2}{|c|}{$\begin{array}{c}\text { Intervention group } \\
(n=115)\end{array}$}} & \multirow{2}{*}{\multicolumn{2}{|c|}{$\begin{array}{c}\text { Comparison group } \\
(n=114)\end{array}$}} & \multirow[b]{3}{*}{$t$-value } & \multirow[b]{3}{*}{ df } & \multirow[b]{3}{*}{$p$-value } \\
\hline & & & & & & & \\
\hline & M & $\mathrm{SD}$ & M & $\mathrm{SD}$ & & & \\
\hline Age (years) & 33.4 & 4.03 & 33.3 & 4.44 & 0.095 & 218 & 0.924 \\
\hline Postpartum days & 3.1 & 1.29 & 2.8 & 1.36 & 1.548 & 227 & 0.123 \\
\hline Attribute & $n$ & $\%$ & $n$ & $\%$ & total & $x^{2}$ & $p$-value \\
\hline \multicolumn{8}{|l|}{ Delivery methods } \\
\hline $\begin{array}{l}\text { normal vaginal } \\
\text { caesarean section }\end{array}$ & 72 & 37.4 & 35 & 30.7 & $\begin{array}{l}151 \\
78\end{array}$ & 1.141 & 0.286 \\
\hline \multicolumn{8}{|l|}{ Birth experience } \\
\hline primipara & 76 & 66.1 & 64 & 56.1 & 140 & & \\
\hline multipara (2 times) & 31 & 27.0 & 40 & 35.1 & 71 & \multirow{3}{*}{2.415} & \multirow{3}{*}{0.491} \\
\hline multipara (3 times) & 7 & 6.1 & 9 & 7.9 & 16 & & \\
\hline multipara (4 times) & 1 & 0.9 & 1 & 0.9 & 2 & & \\
\hline \multicolumn{8}{|l|}{ Previous disease } \\
\hline yes & 108 & 93.9 & 104 & 91.2 & $\begin{array}{c}17 \\
212\end{array}$ & 0.601 & 0.438 \\
\hline \multicolumn{8}{|l|}{ Pregnancy complication } \\
\hline yes & 39 & 66.1 & 29 & 74.6 & 161 & 1.969 & 0.161 \\
\hline \multicolumn{8}{|l|}{ Selected essential oil } \\
\hline citron & 23 & 20.0 & & & & & \\
\hline ylang-ylang & 20 & 17.4 & & & & & \\
\hline pure lavender & 19 & 16.5 & & & & & \\
\hline rosewood & 10 & 8.7 & & & & & \\
\hline Each scale and subscale & M & $\mathrm{SD}$ & M & $\mathrm{SD}$ & $t$-value & df & $p$-value \\
\hline Fatigue & 8.7 & 6.6 & 8.6 & 7.5 & 0.096 & 227 & 0.924 \\
\hline Relaxation & 22.7 & 8.4 & 22.9 & 9.0 & -0.105 & 227 & 0.917 \\
\hline
\end{tabular}

Table 2. Two-way factorial ANOVA with time and intervention in each scale $(\mathrm{N}=229)$.

\begin{tabular}{cccccccc}
\hline Scale & & SS & df & MS & F & \multicolumn{2}{c}{$p$-value } \\
\hline Fatigue & time & 60.97 & 1 & 60.97 & 1.65 & 0.200 & \\
& intervention & 115.76 & 1 & 115.76 & 1.17 & 0.280 & \\
& time $\times$ intervention & 137.19 & 1 & 137.19 & 3.71 & 0.055 & \\
Relaxation & time & 306.41 & 1 & 306.41 & 5.53 & $<0.05$ & $*$ \\
& intervention & 2078.75 & 1 & 2078.75 & 16.81 & $<0.001$ & $* * *$ \\
& time $\times$ intervention & 2198.08 & 1 & 2198.08 & 39.68 & $<0.001$ & $* * *$ \\
\hline
\end{tabular}

${ }^{*} \mathrm{p}<0.05,{ }^{* * *} \mathrm{p}<0.001$. 


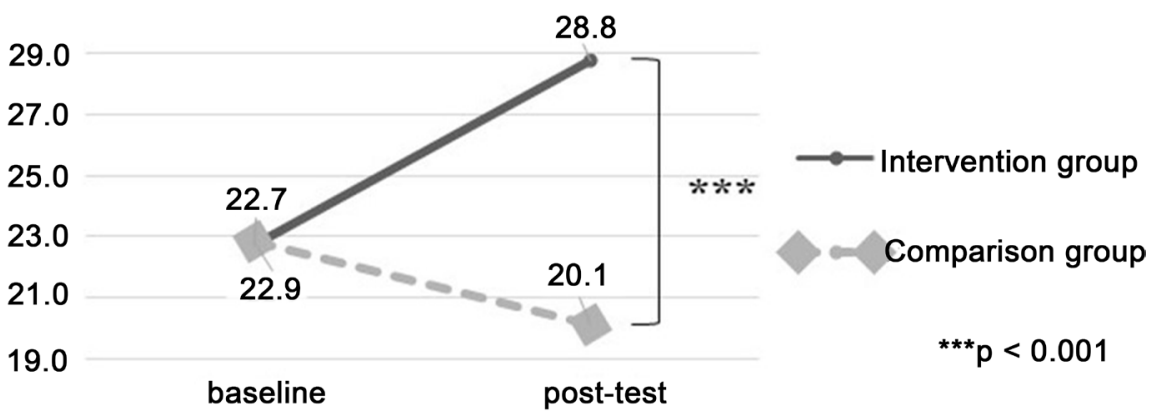

Figure 2. Effects of aroma hand treatment on average relaxation scale score $(\mathrm{N}=229)$.

Fatigue. Therefore, the aroma hand treatment caused a significant increase in relaxation but it did not significantly alleviate fatigue.

\subsection{Process Evaluation}

In the process evaluation, $84.4 \%$ of the participants were satisfied with the treatment methods and $90.5 \%$ felt that the intervention time was appropriate. The majority $(66.1 \%)$ of the participants indicated a high match between their expectations and the intervention, and $91.3 \%$ highly evaluated the provider's communication skills (Figure 3). The results of the content analysis of the open-ended responses revealed 5 categories: 1) increased relaxation, 2) improved physical condition, 3) high satisfaction, 4) need improvement of techniques, and 5) need to maintain an effective amount of essential oil. Comments included both affirmative opinions and suggestions for future improvements (Table 3).

\section{Discussion}

This was a quasi-experimental study wherein data were collected from 2 groups at different times. To verify whether a simple aroma hand treatment is effective in relieving fatigue and promoting relaxation of postpartum mothers, pilot studies were conducted to thoroughly examine this methodology. The relaxation scale scores showed a significant increase in the intervention group compared with the comparison group, but the fatigue scale scores showed no significant decrease. These results suggest that aroma hand treatment using essential oils is effective for promoting relaxation and is highly satisfying for early postpartum mothers.

\subsection{Effectiveness of Aroma Hand Treatment}

Most mothers experience poor sleep and fatigue during the early postpartum period [23]. Maternity blues occur more often on postpartum day 3, and emotional changes associated with childbirth occur on postpartum day 5 until the end of the postpartum period [24]. Therefore, to ensure a good quality sleep for postpartum mothers, it is necessary to alleviate fatigue and promote relaxation.

As mothers in the early postpartum period require care for fatigue reduction and relaxation, it would be beneficial to disseminate aromatherapy treatment as 


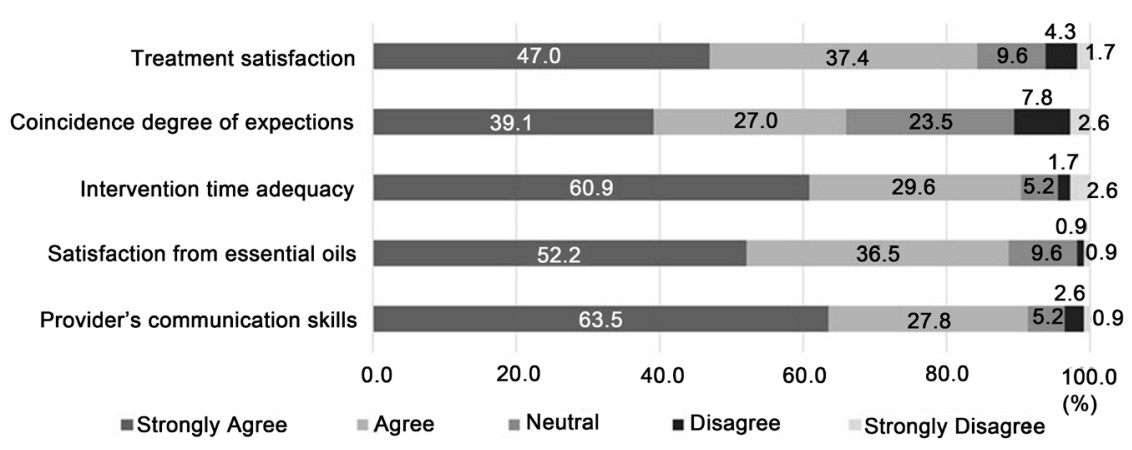

Figure 3. Process evaluation for the intervention group $(n=115)$.

Table 3. Opinions regarding the aroma hand treatment $(n=104)$.

\begin{tabular}{ll}
\hline \multicolumn{1}{c}{ Category } & \\
\hline Increased relaxation & It felt good \\
& I became sleepy \\
& I felt healed \\
Improvement in physical condition & Improvement of edema \\
& Shoulder tensions abated \\
High satisfaction & Improvement of rough hand \\
& I was happy \\
& I was able to select the scent \\
& Experienced at the hospital \\
& Just a good time \\
Need improvement of techniques & Lack of pressure \\
Lack of rhythm \\
Need to maintain an effective amount of & Lack of good scent \\
essential oil & Hope the essential oil is appropriate for the symptoms \\
\hline
\end{tabular}

an integrated component of nursing care [25]. Aromatherapy treatment is part of the nursing holistic care that can be easily administered and therefore has a great advantage. The healing care from aromatherapy has shown a trend of reducing fatigue [26]. In their systematic review, Sánchez-Vidaña et al. indicated that aromatherapy can potentially be an effective therapeutic option for relieving depressive symptoms, with more beneficial effects from aromatherapy massage [27]. In the present study, although fatigue was not reduced, the aroma hand treatment promoted relaxation.

The process evaluation largely received high satisfaction ratings from the participants. However, the participants opined that technical improvements are needed because of the insufficient treatment pressure and rhythm shortage. Further points for improvement included the proper use of essential oils to avoid aroma shortage and the correct choice of the essential oil to relieve particular symptoms. 
Additional studies on how to promote the use of hand aroma treatment as an intervention treatment is recommended because of the ease and rapidity of its application (i.e., 20 minutes) in hospitalized puerperal mothers. Once nurses master the aroma treatment techniques, the process becomes more convenient and easier. Appropriate training and experience of nurses in aromatherapy massage is crucial in achieving positive results [28]. These points for improvement should be taken into consideration in future interventions. In this context, the implementation of an aromatherapy intervention program in many facilities could increase the motivation of postpartum mothers.

\subsection{Limitations and Future Challenges}

There are some limitations of this study. First, the internal validity for the intervention effect was deemed weaker because the research study design was a nonequivalent control group design instead of a randomized controlled trial. Second, limited generalizability and potential bias cannot be completely controlled because data were collected only from a single hospital. Third, it was not possible to measure the duration of the intervention effect because the post-test was conducted only once. Future prospects include training nurses in other maternity hospitals on how to implement aromatherapy treatment. Notably, the nonreduction of fatigue deserves clarification. Moreover, the effectiveness of aroma hand treatment needs to be further analyzed in a larger population before clinical application.

\section{Conclusions}

This study, which clarified the effectiveness of aromatherapy treatment in alleviating fatigue and promoting relaxation of mothers during the early postpartum period, obtained the following important findings:

1) The post intervention fatigue scale scores of the intervention group were not significantly higher than those of the comparison group.

2) The post intervention relaxation scale scores of the intervention group were significantly higher than those of the comparison group.

3) The majority of the participants indicated a high satisfaction with the aroma hand treatment and evaluated the intervention duration as appropriate.

4) Five categories of opinions were extracted from the participants: a) increased relaxation, b) improved physical condition, c) high satisfaction, d) need improvement of techniques, and e) need to maintain an effective amount of the essential oil.

\section{Acknowledgements}

The authors would like to thank all the participants, as well as the facility staff and research assistants for their valuable contributions in this study. The authors also thank Dr. Edward Barroga (http://orcid.org/0000-0002-8920-2607), Advisor of the Academic Writing Desk of St. Luke's International University, Japan, and 
Associate Professor and Senior Medical Editor of Tokyo Medical University, Japan for reviewing and editing the manuscript.

\section{Presentations}

The results of this study were presented at the 37th Annual Conference of the Japan Academy of Nursing Science in 2017.

\section{Conflicts of Interest}

The authors declare that there they have no conflicts of interest associated with this study.

\section{Disclosures}

\section{Human Rights Statements and Informed Consent}

All procedures were performed in accordance with the ethical standards of the responsible committees for human experimentation (institutional and national) and the 1964 Helsinki Declaration and later amendments. Informed consent was obtained from all patients.

\section{Animal Studies}

Animal studies were not performed in this study.

\section{Approval by Ethics Committee}

The protocol of this research project was approved by the Ethics Committee for Epidemiological Studies of Tokyo Healthcare University, Tokyo, Japan (approval no. 27-27) on December 28, 2015.

\section{References}

[1] Darvill, R., Skirton, H. and Farrand, P. (2010) Psychological Factors That Impact on Women's Experiences of First-Time Motherhood: A Qualitative Study of the Transition. Midwifery, 26, 357-366. https://doi.org/10.1016/j.midw.2008.07.006

[2] Hunter, L.P., Rychnovsky, J.D. and Yount, S.M. (2009) A Selective Review of Maternal Sleep Characteristics in the Postpartum Period. Journal of Obstetric, Gynecologic, \& Neonatal Nursing, 38, 60-68. https://doi.org/10.1111/j.1552-6909.2008.00309.x

[3] Cheng, C.Y. and Li, Q. (2008) Integrative Review of Research on General Health Status and Prevalence of Common Physical Health Conditions of Women after Childbirth. Women's Health Issues, 18, 267-280.

https://doi.org/10.1016/j.whi.2008.02.004

[4] Taylor, J. and Johnson, M. (2010) How Women Manage Fatigue after Childbirth. Midwifery, 26, 367-375. https://doi.org/10.1016/j.midw.2008.07.004

[5] Khayamim, N., Bahadoran, P. and Mehrabi T. (2016) Relationship between Fatigue and Sleepiness with General Health of Mothers in the Postpartum Period. Iranian Journal of Nursing and Midwifery Research, 21, 385-390. https://doi.org/10.4103/1735-9066.185580

[6] Bozoky, I. and Corwin, E.J. (2002) Fatigue as a Predictor of Postpartum Depression. 
Journal of Obstetric, Gynecologic, \& Neonatal Nursing, 31, 436-443. https://doi.org/10.1111/j.1552-6909.2002.tb00066.x

[7] Imanishi, J. (2004) Medical Aromatherapy. Japanese Journal of Complementary \& Alternative Medicine, 1, 53-61. (In Japanese) https://doi.org/10.1625/jcam.1.53

[8] Rashidi Fakari, F., Tabatabaeichehr, M., Kamali, H., Rashidi Fakari, F. and Naseri, M. (2015) Effect of Inhalation of Aroma of Geranium Essence on Anxiety and Physiological Parameters during First Stage of Labor in Nulliparous Women: A Randomized Clinical Trial. Journal of Caring Sciences, 4, 135-141.

https://doi.org/10.15171/jcs.2015.014

[9] Fu, C.Y., Moyle, W. and Cooke, M. (2013) A Randomized Controlled Trial of the Use of Aromatherapy and Hand Massage to Reduce Disruptive Behavior in People with Dementia. BMC Complementary \& Alternative Medicine, 13, 165.

https://doi.org/10.1186/1472-6882-13-165

[10] Imura, M., Misao, H. and Ushijima, H. (2006) The Psychological Effects of Aromatherapy Massage in Healthy Postpartum Mothers. Journal of Midwifery \& Women's Health, 51, e21-e27. https://doi.org/10.1016/j.jmwh.2005.08.009

[11] Jahdi, F., Mehrabadi, M., Mortazavi, F. and Haghani, H. (2016) The Effect of Slow-Stroke Back Massage on the Anxiety Levels of Iranian Women on the First Postpartum Day. Iranian Red Crescent Medical Journal, 18, e34270. https://doi.org/10.5812/ircmj.34270

[12] Asazawa, K., Kato, Y., Yamaguchi, A. and Inoue, A. (2017) The Effect of Aromatherapy Treatment on Fatigue and Relaxation for Mothers during the Early Puerperal Period in Japan: A Pilot Study. International Journal of Community Based Nursing and Midwifery, 5, 365-375.

[13] Behruzi, R., Hatem, M., Fraser, W., Goulet, L., Ii, M. and Misago, C. (2010) Facilitators and Barriers in the Humanization of Childbirth Practice in Japan. BMC Pregnancy and Childbirth, 10, 25. https://doi.org/10.1186/1471-2393-10-25

[14] Cohen, J. (1992) A Power Primer. Psychological Bulletin, 112, 155-159. https://doi.org/10.1037/0033-2909.112.1.155

[15] Tillett, J. and Ames, D. (2010) The Uses of Aromatherapy in Women's Health. The Journal of Perinatal \& Neonatal Nursing, 24, 238-245. https://doi.org/10.1097/JPN.0b013e3181ece75d

[16] Hashemi, S.H., Hajbagheri, A. and Aghajani, M. (2015) The Effect of Massage with Lavender Oil on Restless Leg Syndrome in Hemodialysis Patients: A Randomized Controlled Trial. Nursing and Midwifery Studies, 4, e29617. https://doi.org/10.17795/nmsjournal29617

[17] Nakakita, M. and Takenoue, K. (2009) Effect of Relaxing Back Massages on Early Healthy Postpartum Mothers-Autonomic Nervous System Activity and Subjective Analysis. Journal of Japan Academy of Midwifery, 23, 230-240. (In Japanese) https://doi.org/10.3418/jjam.23.230

[18] International Center for Occupational Safety and Health (2004) Self-Diagnosis Check List for Assessment of Worker's Accumulated Fatigue. JICOSH.

https://www.jniosh.go.jp/icpro/jicosh-old/english/topics/AccumulatedFatigueCheck List/AccumulatedFatigueCheckList.html

[19] Sasaki, T., Iwasaki, K., Mori, I., Hisanaga, N. and Shibata, E. (2007) Overtime, Job Stressors, Sleep/Rest, and Fatigue of Japanese Workers in a Company. Industrial Health, 45, 237-246. https://doi.org/10.2486/indhealth.45.237

[20] Nedate, K. and Kamisato, I. (1984) Effects of Cognition of Physiological State and Actual Physiological Responses upon Emotion. Japanese Association of Behavioral 
and Cognitive Therapies, 9, 33-39. (In Japanese)

[21] Takahashi, M. (1996) The Effects of Relaxation on Guided Imagery of Pregnant Women and Fetuses. Kyorin University, Tokyo. https://kaken.nii.ac.jp/ja/grant/KAKENHI-PROJECT-06807183/

[22] Lyn, R. (2015) Handling Qualitative Data: A Practical Guide. 3rd Edition, Sage, Newcastle upon Tyne, 67-122.

[23] Lee, S.Y. and Lee, K.A. (2007) Early Postpartum Sleep and Fatigue for Mothers after Cesarean Delivery Compared with Vaginal Delivery: An Exploratory Study. The Journal of Perinatal \& Neonatal Nursing, 21, 109-113. https://doi.org/10.1097/01.JPN.0000270627.73993.b0

[24] Gonidakis, F., Rabavilas, A.D., Varsou, E., Kreatsas, G. and Christodoulou, G.N. (2007) Maternity Blues in Athens, Greece: A Study during the First 3 Days after Delivery. Journal of Affective Disorders, 99, 107-115. https://doi.org/10.1016/j.jad.2006.08.028

[25] Smith, M.C. and Kyle, L. (2008) Holistic Foundations of Aromatherapy for Nursing. Holistic Nursing Practice, 22, 3-9. https://doi.org/10.1097/01.HNP.0000306321.03590.32

[26] Murakami, A., Kita, S. and Kamiya, K. (2008) Effect of Iyashi Healing Care in Early Puerperium on Mother's Fatigue and Breastfeeding. Journal of Japan Academy of Midwifery, 22, 136-145. (In Japanese) https://doi.org/10.3418/jjam.22.136

[27] Sánchez-Vidaña, D.I., Ngai, S.P., He, W., Chow, J.K., Lau, B.W. and Tsang, H.W. (2017) The Effectiveness of Aromatherapy for Depressive Symptoms: A Systematic Review. Evidence-Based Complementary and Alternative Medicine, 2017, Article ID: 5869315.

[28] Gok Metin, Z., Arikan Donmez, A., Izgu, N., Ozdemir, L. and Arslan, I.E. (2017) Aromatherapy Massage for Neuropathic Pain and Quality of Life in Diabetic Patients. Journal of Nursing Scholarship, 49, 379-388.

https://doi.org/10.1111/jnu.12300 